\title{
Multiple domains of the RNA polymerase $I$ activator hUBF interact with the TATA-binding protein complex hSL1 to mediate transcription
}

\author{
Hans-Michael Jantzen, ${ }^{1}$ Alice Ming Chow, ${ }^{2}$ David S. King, and Robert Tiian \\ Howard Hughes Medical Institute, Department of Molecular and Cell Biology, Division of Biochemistry and Molecular \\ Biology, University of California at Berkeley, Berkeley, California 94720 USA
}

\begin{abstract}
Recent evidence suggests that transcription initiation by all three eukaryotic RNA polymerases involves a complex of the TATA-binding protein (TBP) and multiple TBP-associated factors (TAFs). Here, we map the functional domains of the nucleolar HMG box protein hUBF, which binds to the human rRNA promoter and stimulates transcription by RNA polymerase I through cooperative interactions with a distinct TBP-TAF complex, hSL1. DNase I footprint analysis of mutant hUBF proteins and of a synthetic peptide of 84 amino acids reveals that HMG box 1 is necessary and sufficient for DNA sequence specificity, whereas other HMG boxes and the amino terminus modulate the binding efficiency. hUBF contains multiple activation domains that include the acidic carboxyl terminus and three HMG boxes. HMG boxes 3 and 4 and the acidic tail contribute significantly to an extended footprinting pattern in the presence of hSL1, suggestive of specific protein-protein interactions. Moreover, the inability of XUBF from Xenopus laevis to form an initiation complex with hSL1 can be overcome by hybrid proteins containing human HMG box 4 and the acidic carboxyl terminus. These results strongly suggest an important role of transcription activation domains of hUBF in mediating interactions with the TBP-TAF complex hSL1.
\end{abstract}

[Key Words: UBF; HMG box; SLl; protein-protein interaction; TATA-binding protein; rRNA transcription]

Received June 30, 1992; revised version accepted July 30, 1992.

In recent years considerable progress has been made toward elucidating the molecular mechanisms of transcription initiation. In particular, a multitude of sequence-specific transcription factors that bind to cis-acting promoter and enhancer elements have been identified and their structure and function have been analyzed (Johnson and McKnight 1989; Mitchell and Tiian 1989). Moreover, a number of components of the basal transcriptional apparatus have been implicated as targets for activation (Sawadogo and Sentenac 1990; Greenblatt 1991; Roeder 1991; Pugh and Tjian 1992). Although domains involved in transcriptional activation by sequence-specific transcription factors have been identified, their targets in the basal transcriptional machinery remained elusive.

The recent identification of coactivators and TATAbinding protein-associated factors (TAFs) that are associated with the TATA-binding protein $(T B P \mid$ in the TFIID fraction of the RNA polymerase II (Pol II) system focused our attention on a novel class of transcription

Present addresses: ${ }^{1}$ COR Therapeutics, Inc., South San Francisco California 94080 USA; ${ }^{2}$ Genentech, Inc., Department of Cardiovascular Research, South San Francisco California 94080 USA. factors. At least some of these TAFs are required for activation by sequence-specific regulatory proteins (Pugh and Tjian 1990, 1991; Dynlacht et al. 1991; Tanese et al. 1991; Gill and Tjian 1992). Thus, there appear to be adaptor molecules that link the basal transcriptional apparatus to the activators. Several recent reports indicate that TBP is not only involved in transcription initiation by RNA Pol II but is also necessary for transcription by RNA polymerase III (Pol III) as well as RNA polymerase I (Pol I) (Lobo et al. 1991; Margottin et al. 1991; Simmen et al. 1991; Comai et al. 1992; Cormack and Struhl 1992; Schultz et al. 1992; White et al. 1992). These studies strongly support the unifying principle that in spite of vast differences in specifity, promoter recognition, and subcellular localization of the three polymerases, TBP constitutes an integral component of the transcription initiation complex in all three systems. We hypothesized that in each system, TBP could be decorated with distinct sets of TAFs that would direct specificity with respect to type of RNA polymerase, initiation site, and class of activators. These distinct TBP-TAF complexes could also serve as coactivators that are responsible for mediating activation by site-specific transcription regulators. 
Our previous studies revealed that transcription of vertebrate ribosomal RNA (rRNA) by RNA Pol I requires at least two additional transcription factors: (1) the promoter selectivity factor $\mathrm{SL} 1$ and (2) the upstream-binding factor (UBF) (Learned et al. 1986; Bell et al. 1988, 1990; Reeder 1990; Sollner-Webb and Mougey 1991). Human (h)UBF is a sequence-specific DNA-binding protein that interacts with both the core and upstream control element (UCE) of the human rRNA promoter, whereas hSLl alone cannot bind site specifically to the DNA. Instead, in the presence of hUBF, hSLl forms a complex on the human promoter that is necessary for transcriptional activation. The cooperative binding of UBF and SLl to the promoter is demonstrated most clearly by an enhanced DNase I footprint that, in the presence of both proteins, is extended into the UCE region of the promoter critical for transcriptional activation (Learned et al. 1986; Bell et al. 1988). The potential direct interaction between SLl and UBF provides a unique opportunity to study in detail the mechanism of activation by a sitespecific transcription factor.

The transcriptional activator UBF has been purified to homogeneity from human, rodent, and frog cells (Bell et al. 1988, 1990; Dunaway 1989; Pikaard et al. 1989; Smith et al. 1990). Molecular cloning of the $97-\mathrm{kD}$ form of the 94/97-kD doublet of hUBF (Jantzen et al. 1990) revealed at least four repeated regions of 80-90 amino acids with sequence homology to the nonspecific DNAbinding domains of high mobility group proteins 1 and 2 (HMG 1 and 2), which have been termed HMG boxes. Initial studies demonstrated that a hUBF deletion mutant consisting of HMG box 1 and $\sim 100$ amino acids of the amino terminus was sufficient to bind specifically to the human rRNA promoter in a column-binding assay establishing that the HMG box is a novel sequence-specific DNA-binding motif (Jantzen et al. 1990). Since the initial characterization of the HMG boxes of hUBF, a superfamily of HMG box proteins has emerged, including, among others, mitochondrial factors ABF2 and mtTF1 (Diffley and Stillman 1991; Parisi and Clayton 1991), tissue-specific transcription factors TCF-1 $\alpha /$ LEF-1 and TCF-1 (Travis et al. 1991; van de Wetering et al. 1991; Waterman et al. 1991), and the mammalian testis-determination factor SRY (Gubbay et al. 1990; Sinclair et al. 1990). hUBF is unique in having more than two HMG boxes and, as with HMG 1 and 2, a very acidic carboxyl terminus $(64 \%$ acidic amino acids), which we proposed might play a role in the interaction with the transcription factor hSL1. These findings suggest that hUBF may represent a class of regulators that lies somewhere between highly site-specific transcriptional activators, such as TCF-1, and the more abundant chromosomal proteins, such as HMG 1, 2, and histone H1.

Recently, the $94-\mathrm{kD}$ form of hUBF was cloned and shown to contain a 37-amino-acid deletion within HMG box 2, apparently owing to differential splicing /Chan et al. 1991; O'Mahoney and Rothblum 1991). Whereas mammalian UBFs appear to be structurally and functionally closely related (Hisatake et al. 1991; O'Mahoney and Rothblum 1991; B. Drees, H.-M. Jantzen, and R. Tjian, unpubl.), xUBF from Xenopus laevis displays some interesting differences. For example, both forms of xUBF have sustained a deletion of HMG box 4, although otherwise their sequences are highly similar to hUBF (Bachvarov and Moss 1991; Bachvarov et al. 1991; McStay et al. 1991a; A.M. Chow, C. Altmann, H.-M. Jantzen, and R. Tjian, unpubl.). Curiously, xUBF binds to the same DNA sequences that are recognized by hUBF (Bell et al. 1989; Pikaard et al. 1989). However, xUBF is a potent activator of $X$. laevis Pol I but not of human Pol I. One intriguing possibility is that a crucial protein-protein interaction with human hSLl has been disrupted owing to the inherent structural differences between xUBF and hUBF (Bell et al. 1989; Pikaard et al. 1989). It should therefore be feasible to dissect the structural basis of the species specificity of transcriptional activation mediated by UBF and to determine its interaction with SLl. Recently, the importance of the three HMG boxes, the acidic tail, and the amino terminus of xUBF for transcriptional activation of RNA Pol I from $X$. laevis was reported (McStay et al. 1991b). The finding that purified hSLl is actually composed of the TATA-binding protein and three distinct TAFs (Comai et al. 1992), together with the availability of cloned hUBF and $\mathrm{xUBF}$, prompted us to focus our analysis on the role of activation domains and their potential interaction with the TBP-TAF initiation complex.

In this paper we delineate the functional domains of hUBF, with particular emphasis on the differential roles of the multiple HMG boxes. First, we mapped the regions of the protein required for sequence-specific interaction with the human rRNA promoter. We then addressed the relationship of hUBF domains involved in transcriptional activation and their potential interaction with the TATA-binding protein complex hSL1. Finally, we took advantage of the species-specific activation function of $x U B F$ from $X$. laevis to demonstrate specificity and the link between the TBP-TAF complex and specific activation domains of hUBF. Our results provide the first evidence that activation domains of transcriptional regulators such as hUBF may promote initation by interacting with components of the TBP-TAF complex.

\section{Results}

HMG box 1 of hUBF is necessary and sufficient for specific DNA binding

DNA binding of hUBF to the human rRNA promoter is altered dramatically by the TBP-TAF complex hSLl, and cooperative interactions between the two factors are required for transcriptional activation. Therefore, as a first step toward characterizing the functional domains of hUBF, we have determined the portions of the protein involved in DNA binding. Previously, we demonstrated a role for HMG box 1 of hUBF in site-specific DNA binding in a DNA column-binding assay (Jantzen et al. 1990). In the case of $x U B F$, the presence of any one of the three HMG boxes has been found to be sufficient for binding to the $X$. laevis rRNA promoter using a similar assay (Mc- 
Stay et al. 1991b). The contribution of the multiple HMG boxes to specific protein-DNA interactions, however, had not been defined directly in a DNase I footprinting assay. Therefore, we have expressed a number of carboxy-terminal, amino-terminal, and internal deletions of hUBF by translation in vitro (see Fig. 4C, below) and partially purified the proteins by DNA affinity chromatography. Equivalent amounts of each mutant protein were titrated in a DNase I protection assay using the noncoding strand of the human rRNA promoter. Successive removal of the carboxyl terminus and HMG box 4 did not change DNA-binding specificity or efficiency (Fig. 1A, lanes 1-16). In contrast, deletion of HMG box 3, and boxes 3 and 2 led to a 4 - or 16-fold reduction of binding efficiency, respectively (lanes 17-26). These data suggest that the amino-terminal 204 amino acids of hUBF display the same DNA-binding specificity as the full-length protein and that HMG boxes 2 and 3 contribute to binding efficiency. When the carboxy-terminal one-third of HMG box 1 was deleted (mutant 154C), specific binding was reduced $\geqslant 400$-fold (lane 28 ) when compared with the full-length protein, demonstrating that the integrity of this DNA-binding motif is required for specific binding.

Removal of the amino terminal 83 amino acids (mutant $84 \mathrm{~N}$ ) resulted in essentially the loss of footprinting activity even with a 235 -fold excess of protein (Fig. 1A, lane 31). No specific DNA-binding activity could be detected with a 25 -fold excess of mutants $182 \mathrm{~N}$ and $284 \mathrm{~N}$, which lack HMG boxes 1 and 2 , respectively (lanes $33,35)$. This suggests an important contribution of the amino terminus in the site-specific DNA binding of hUBF to the human rRNA promoter.

The role for each individual HMG box in specific DNA binding was investigated with internal deletions (Fig. 1B). Mutants lacking box 2 or 4 behaved essentially as wild-type hUBF (lanes 9-11,22-24). Removal of HMG box 3 (Fig. 1B, mutant db3) or of the region between HMG box 4 and the acidic tail (Fig. 1B, mutant dx) reduced binding about fourfold (lanes 19-22,26-28). In stark contrast, footprinting activity was reduced $\geqslant 100$ fold in the absence of HMG box 1 (Fig. 1B, lane 6; mutant $\mathrm{db} 1)$. Although hUBF mutants lacking either the amino terminus or HMG box 1 do not display any specific promoter binding in a DNase I footprinting assay, mutant proteins containing at least one HMG box can still bind to DNA columns bearing UBF recognition sequences in the presence of nonspecific competitor (data not shown; see Fig. 4C). Thus, HMG boxes $2-4$ are DNA-binding domains on their own, however, with no detectable specificity in a more stringent assay system.

These results strongly suggest a dominant role for both HMG box 1 and the amino terminal domain in specific and efficient DNA binding by hUBF. We have addressed the contribution of HMG box 1 directly by synthesizing an 84-amino-acid peptide encompassing residues 103186 of hUBF. Amino terminal truncations of HMG box 1 were generated by terminating the peptide synthesis after 75 or 65 amino acids. When analyzed for specific DNA binding on the human rRNA promoter using
DNase I footprinting, the 84-mer protected the promoter from DNase I digestion in a manner very similar to wildtype hUBF. The specific activity of the peptide was significantly reduced, however, relative to the full-length protein (Fig. 1C, lanes 5-7). In addition, the characteristic enhanced band in the center of the UCE footprint was less pronounced and the weak enhanced cleavage site in the core promoter was no longer detectable. The truncated peptides (Pep75 and Pep65) displayed an increasingly relaxed binding specificity and a substantially reduced binding efficiency (lanes 9-16).

Taken together these data demonstrate that HMG box 1 is necessary and sufficient for specific DNA binding by hUBF. Both the amino terminus and the other HMG boxes that lack detectable specific DNA binding by themselves, however, appear to enhance binding by HMG box 1 .

\section{The amino terminus has the potential to form dimers}

We have reported previously that the amino terminus of hUBF has no detectable DNA-binding activity on its own (Jantzen et al. 1990). DNase I footprinting analysis of deletion mutants and HMG box 1 peptides presented here, however, reveal a substantial drop in specific DNAbinding activity in the absence of the amino terminus. One possible mechanism by which non-DNA-binding domains can contribute to DNA-binding efficiency is dimerization. The amino terminus of $x U B F$ has been shown to contain a bipartite dimerization domain (McStay et al. 1991b). Here, we have analyzed the ability of full-length hUBF, as well as two critical deletion mutants $(204 \mathrm{C}, 84 \mathrm{~N})$, to form dimers in the presence of the chemical cross-linking reagent EGS. In the presence of EGS the wild-type protein was completely converted to a dimer species migrating on an SDS gel at $\sim 200 \mathrm{kD}$ (Fig. $2 \mathrm{~A}$, lanes 1,2 ). The mutant encompassing only HMG box 1 and the amino terminus (204C) was also efficiently converted into dimers (lanes 3,4$)$. In contrast, when the amino-terminal 83 amino acids were deleted (mutant $84 \mathrm{~N}$ ), dimer formation was lost and the protein band became diffuse probably owing to intramolecular crosslinked species (lanes 5,6$)$. These data suggest that the amino terminal domain contributes to the DNA-binding activity of hUBF by forming dimers that bind DNA with higher efficiency.

The transcriptional activation domain of hUBF
is a composite of $H M G$ boxes and the acidic tail

To map activation domains of hUBF, we have used in vitro-translated and affinity-purified mutant proteins to reconstitute transcription with hSLl and Pol I. We have analyzed a panel of carboxy- and amino-terminal mutants (Fig. 3A), as well as internal deletions (Fig. 3B), over a range of protein concentrations for transcriptional activity in vitro. Transcriptional activation was reduced dramatically (from 13-to 24-fold stimulation for the wild type to 1.4- to 1.7-fold activation) when the carboxyterminal acidic tail of hUBF was removed (mutants $670 \mathrm{C}$ 

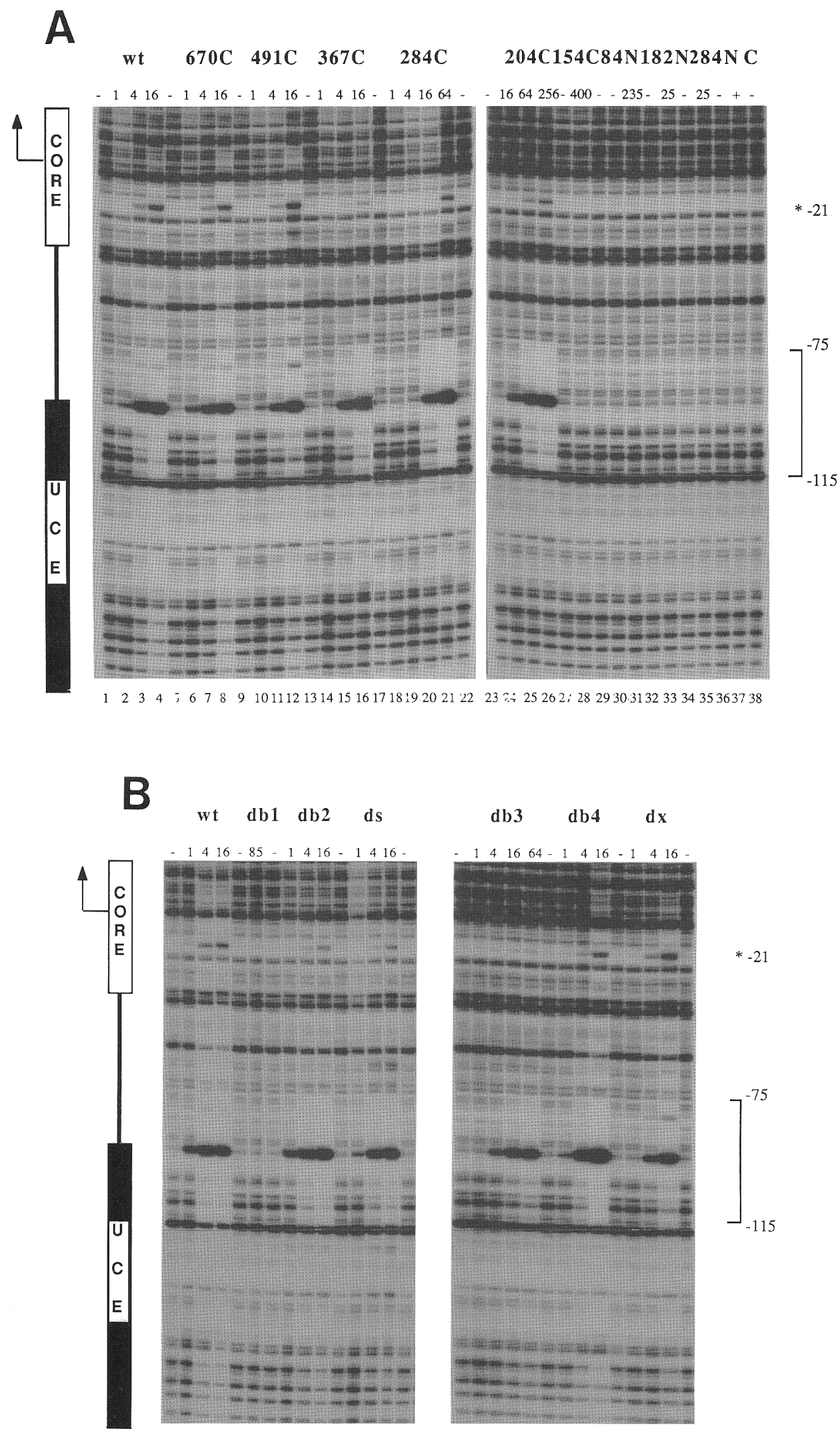

db3 db4 dx

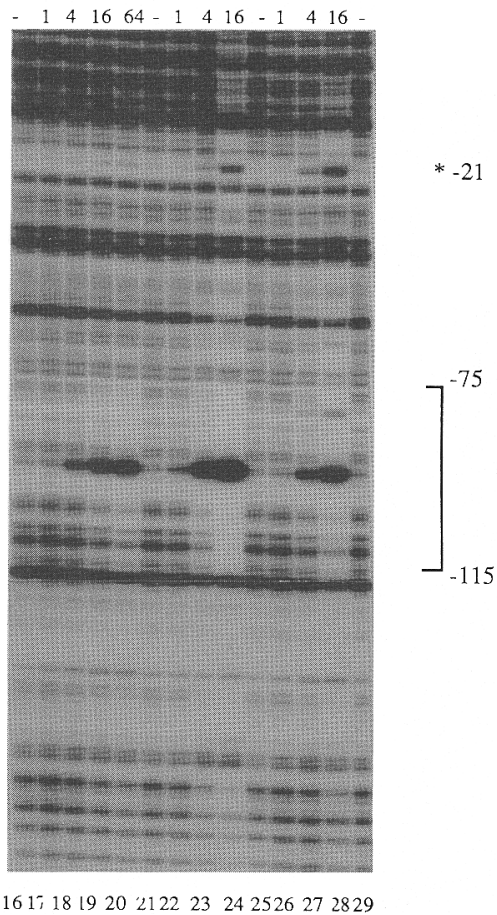


and 491C). Mutants lacking the acidic tail and HMG box 4 (367C-204C) were completely inactive for transcription. Mutants 670C, 491C, 367C, and 204C have also been expressed and purified from recombinant vaccinia viruses, and very similar results were obtained (H.-M. Jantzen, unpubl.). Deletion of the amino-terminal 83 amino acids that significantly reduced footprinting activity (see Fig. 1A) also diminished transcriptional activation (Fig. 3A, lanes 21,22). Internal deletion of $\mathrm{HMG}$ box 2 or 4 , leaving the remainder of hUBF intact, reduced transcriptional activation dramatically (from 13-fold to 2- to 3-fold; Fig. 3B, mutants db2 and db4), and removal of HMG box 3 essentially eliminated transcriptional activation (Fig 3B, mutant db3). Mutant dx, (Fig. 3B), in which the region between the carboxyl terminus and the HMG box 4 had been removed, was only slightly reduced in transcriptional activation (to eightfold). Surprisingly, mutants that lack HMG box 1 or the amino terminus and display no detectable specific DNA-binding on their own (mutants dbl, $84 \mathrm{~N}, 182 \mathrm{~N}$ ) still retain some transcriptional activity. This suggests that in contrast to the footprinting reaction /which contains only hUBF and DNA), other components of the in vitro transcription system (such as SL1) might contribute to the specificity and stability of DNA binding of the complex to the promoter, thus diminishing the requirement for the amino terminus or the HMG box 1. Taken together, our data suggest that the most dramatic contributions to tran- scriptional activation by hUBF derive from HMG boxes 2,3 , and 4 and the acidic tail. In contrast to the results presented here, the amino terminus of xUBF contains a strong transcriptional activation domain independent of its dimerization function, and the acidic carboxyl terminus is much less important than in hUBF (McStay et al. 1991b). This indicates some critical differences in the function of $x U B F$ which, despite its extensive homology with hUBF, cannot replace hUBF to activate human RNA Pol I transcription.

\section{hUBF domains required for cooperative DNA binding of $h S L 1$}

We have hypothesized that transcriptional activation of RNA Pol I by hUBF requires specific protein-protein interactions with hSLl (Learned et al. 1986; Bell et al. $1988,1989,1990)$. For example, in the presence of hSL1, the hUBF footprint is enhanced and extended into the UCE region of the human $\mathrm{RNA}$ promoter most critical for hUBF function (Learned et al. 1986; Bell et al. 1988). Recently, we demonstrated that purified hSL1 is a multisubunit complex consisting of the TBP and three distinct TAFs (Comai et al. 1992). Having identified the transcriptional activation domains of hUBF, we are now in a good position to test the idea that these same domains are responsible for cooperative DNA binding of hSLl. This line of experiments also provides a unique

Figure 1. HMG box 1 and the amino terminus of hUBF are required for efficient and specific DNA binding. $(A)$ DNase I footprinting analysis of carboxy-terminal and amino-terminal deletions. hUBF deletion mutants (see Fig. 4C) were footprinted on the noncoding strand of the human rRNA promoter. The numbers above the lanes indicate relative molar amounts of the proteins. Lanes 1,5,9,13, $17,22,23,27,29,30,32,34,36$, and 38 show reactions without protein; lane 37 $(\mathrm{CON})$ represents a footprinting reaction containing $20 \mu \mathrm{l}$ of a mock translation reaction (no RNA) passed over a DNA affinity column. The position of the human promoter elements is shown at left. The bracket at right indicates the region of protection overlapping the UCE; the asterisk (*) identifies a position of enhanced cleavage in the core region of the promoter. $(B)$ DNase I footprinting analysis of internal deletions. Internal deletion mutants of hUBF (see Fig. 4C) were footprinted on the noncoding strand of the human rRNA promoter. The conditions used were identical to those described in $A$. $(C)$ An HMG box 1 peptide has a DNA-binding specificity similar to full-length hUBF. Wild-type hUBF purified from HeLa cells (lanes 2,3) or synthetic peptides of 84,75 , or 65 amino acids of HMG box 1 were footprinted on the noncoding strand of the human rRNA promoter. Relative molar amounts of proteins and peptides used are indicated above the lanes and the position of the peptides in the hUBF sequence is given above the brackets.

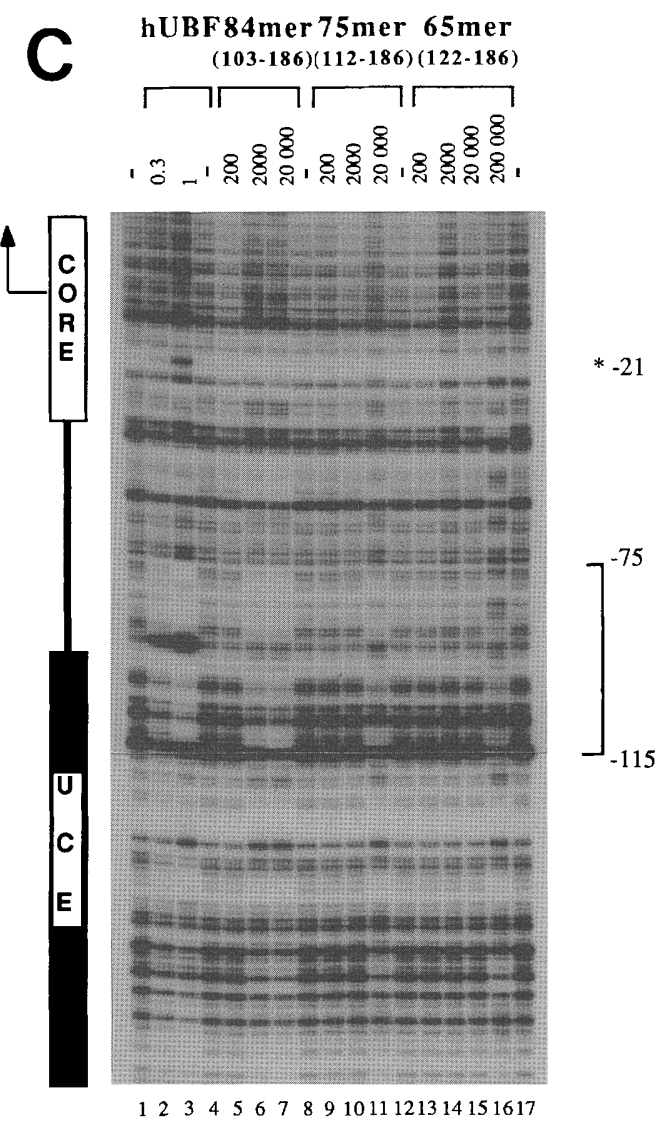


A

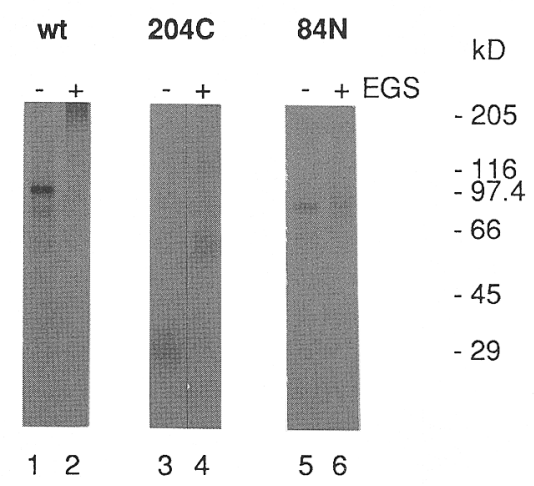

B

box 1 box 2 box 3 box 4

acidic tail

wt

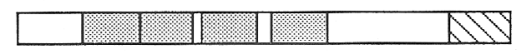

$204 \mathrm{C}$

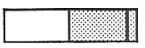

$84 \mathrm{~N}$

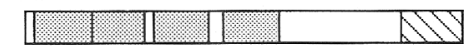

Dimer

Figure 2. The amino terminus of hUBF is required for dimerization. $(A)$ Wild-type hUBF or deletion mutants were translated in vitro incorporating $\left[{ }^{35} S \mid\right.$ methionine and partially purified by DNA affinity chromatography. The proteins were incubated in the absence $(-)$ or presence $(+)$ of EGS cross-linking agent, separated by SDS-PAGE ( $8 \%$ acrylamide), and the gel was autoradiographed. The positions of molecular mass size marker proteins are shown at right (in $\mathrm{kD}) .(B)$ Schematic diagram of the deletion mutants and wild-type hUBF used in the cross-linking experiments shown in $A$. Dimer formation is indicated with $\mathrm{a}+$.

opportunity to investigate the possible role of specific protein-protein interactions between a transcriptional activator and a TBP-TAF complex.

Mutant hUBF proteins were assayed for specific DNAbinding in the absence and presence of partially purified hSLl. The wild-type hUBF protein displayed the characteristic extension of the protected region in the presence of SLl (Fig. 4A, lanes 2,3). Deletion of the acidic tail, which impairs transcriptional activity significantly, generated an altered pattern of the protected region in the presence of hSL1 (Fig. 4A, lanes 5,6; mutant 670C). This altered footprint is also generated with mutant 491C, which consists of the amino terminus and the four HMG boxes (lanes 8,9$)$. Most important, the mutant lacking HMG box $4(367 \mathrm{C})$ no longer displays any extended footprint pattern in the presence of hSLl (lanes 12,13) and, concomitantly, this mutant has completely lost the ability to activate transcription. There was also no footprint extension detectable with mutant $\mathrm{db} 3$ in which only
HMG box 3 had been deleted (Fig. 4B, lanes 18,19). As expected, this mutant was completely inactive in transcription. Removal of either HMG box 4 (mutant db4) or the region between this domain and the acidic tail $(\mathrm{mu}-$ tant $d x$ ) affected footprint extension only partially (Fig. $4 \mathrm{~B}$, lanes 21,22 , and 14,15 , respectivelyl, and transcription was also only partially inhibited but not eliminated. As expected, the 84-mer synthetic peptide spanning HMG box 1 did not display any interaction with hSLl (Fig. 4A, lanes 32,33). In short, all mutants of hUBF impaired for formation of an extended footprint with hSLl also displayed diminished transcriptional activity (Fig. $4 \mathrm{C}$ ). Thus, there is a strong correlation between domains of hUBF involved in transcriptional activation and cooperative DNA binding of hSL1. In experiments not shown here, we have evidence that purified TBP alone is not capable of directing interactions with hUBF and that there is no detectable extended footprint $(\mathrm{H}$.-M. Jantzen, unpubl.). Consequently, it is most likely that HMG boxes 3 and 4 , and the acidic tail interact with one or more TAFs of the hSL1 complex.

\section{Activation by hUBF may also involve interaction with components of the transcriptional machinery other than hSL1}

Thus far, our data suggest that the primary mechanism of activation by hUBF is to interact and perhaps direct cooperative complex formation with hSLl at the promoter. It seems possible, however, that a large polypeptide such as hUBF, with multiple activation domains, might make contact with more than a single target. Consequently, we searched through our panel of mutant hUBF proteins for candidates that might represent lesions in activation that would not necessarily impede hSL1 interactions. We found two cases in which a strict correlation between transcriptional activity and cooperative DNA binding of hSLl was uncoupled. First, internal deletion of HMG box 2 (mutant db2) rendered hUBF deficient in transcriptional activation, but no change was detectable in the formation of an extended footprint with hSL1 (Fig. 3B, lanes 12-14; Fig. 4B, lanes 8,9). This finding suggests that HMG box 2 may contribute activation function by interaction with RNA pol I or involvement in directing an active conformation of the promoter DNA sequences, or both.

A second piece of evidence suggesting the involvement of an activation domain in UBF that is not directly interacting with SLl comes from a natural variant, the 94-kD form of mammalian UBF. Recall that UBF from human or rodent cells consists of a protein doublet of 97 and 94-kD in size (Bell et al. 1988, 1990; Smith et al. 1990). We have observed that the $97-\mathrm{kD}$ form expressed from the cloned CDNA either using a vaccinia virus or an in vitro translation system displays all of the functional properties of the doublet isolated from HeLa cells (this paper; Jantzen et al. 1990). The smaller $94-\mathrm{kD}$ form is encoded by a differentially spliced RNA resulting in a deletion of 37 amino acids within HMG box 2 /Chan et 


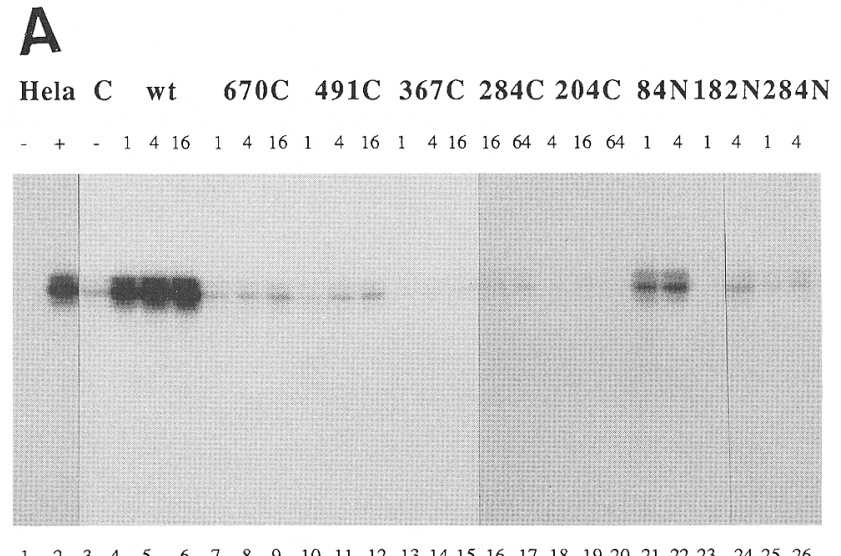

$\begin{array}{llllllllllllllllllllllllll}1 & 2 & 3 & 4 & 5 & 6 & 7 & 8 & 9 & 10 & 11 & 12 & 13 & 14 & 15 & 16 & 17 & 18 & 19 & 20 & 21 & 22 & 23 & 24 & 25 & 26\end{array}$

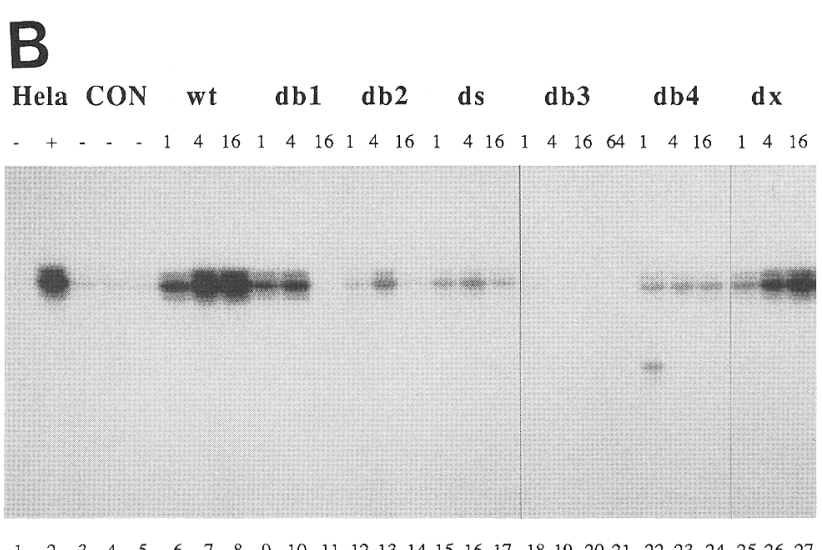

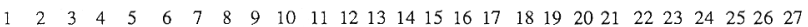

Figure 3. Transcriptional activation in vitro of RNA Pol I by deletion mutants of hUBF. Wild-type or mutant forms of hUBF (see Fig. $4 C$ ) were expressed by translation in vitro and partially purified by affinity chromatography. Various amounts (relative molar amounts indicated by the numbers above the lanes) were tested in an in vitro transcription system consisting of partially purified RNA Pol I and SL1 from HeLa cells. RNA correctly transcribed from a human rRNA promoter template was detected by S1 nuclease analysis. $(A)$ Transcriptional activation by carboxy- and amino-terminal deletion proteins. Lanes 1 and 3 contain no UBF proteins; lane 2 contains $0.5 \mathrm{ng}$ of affinity-purified hUBF from HeLa cells; lane 3 contains $4 \mu \mathrm{l}$ of a mock translation reaction (no RNA) that has been processed over a DNA affinity column in parallel with the hUBF proteins. $(B)$ Transcriptional activation by internal deletion mutants. Experimental conditions were identical to $A$. Lanes $3-5(\mathrm{CON})$ contain 1,2 , and $4 \mu \mathrm{l}$, respectively, of a mock translation reaction passed over a DNA affinity column. The ds mutant represents the $94-\mathrm{kD}$ splicing variant of hUBF.

al. 1991; Hisatake et al. 1991; O'Mahoney and Rothblum 1991). This protein has been shown to bind to UBF recognition sequences in the presence of the large form during affinity purification procedures and in UV cross-linking experiments (S.P. Bell, unpubl.), yet its transcriptional properties have not been characterized. We have expressed the $94-\mathrm{kD}$ form of hUBF [here referred to as ds; the cDNA was a generous gift of Drs. E.K.L. Chan and E.M. Tan (Chan et al. 1991)] by translation in vitro. DNase I footprinting analysis clearly shows that it binds to the human rRNA promoter with the same specificity and efficiency as the $97-\mathrm{kD}$ wild-type form (here referred to as wt; Fig. 1B, cf. lanes 12-14 with lanes 2-4). It is also capable of forming an extended footprint with SL1 indicative of a productive protein-protein interaction with the TBP-TAF complex (Fig. 1B, cf. lanes 11 and 12 with lanes 2 and 3). Interestingly, however, this variant form, which is lacking a portion of HMG box 2, activates transcription very poorly $(2.5$-fold instead of 13 -fold; Fig. 3B, lanes 15-17). These results, which are very similar to those obtained with a complete deletion of HMG box 2, support the critical role of this part of hUBF in transcriptional activation that is independent of interactions with $\mathrm{hSL}$ l. In addition, these findings underscore the unique and nonredundant functional characteristics of the different copies of the HMG box motif that had not been determined until now. These results also reveal that not all mutants defective in transcription are necessarily defective in interaction with hSLl. Instead, some activation domains of hUBF might interact with other components of the initiation complex. Finally, considering the extensive expression of the $94-\mathrm{kD}$ form in most mammalian species tested, we are intrigued by the prospect of potentially important but thus far unknown regulatory functions of this truncated protein.

\section{Rescue of transcriptional activation} by a heterologous UBF with specific domains of hUBF

$\mathrm{xUBF}$ from $X$. laevis has been shown to lack any detectable transcriptional activity when reconstituted with human Pol I and SL1 using the human promoter template, although its DNA-binding specificity is indistinguishable from hUBF. As expected, xUBF does not display a fully extended footprint in the presence of hSLl (Bell et al. 1989; Pikaard et al. 1989). To study the structural basis for this difference between hUBF and xUBF and to use these differences to obtain more insights into the mechanism of transcriptional activation, we have cloned cDNAs for xUBF. One representative clone that has been completely sequenced encodes a protein identical to xUBF1 (Bachvarov and Moss 1991). We also obtained a clone that appears to be identical to the xUBF2 form encoded by a different gene (Bachvarov et al. 1991; McStay et al. 1991a). The amino acid sequence of xUBF is remarkably similar to hUBF (Fig. 5A): within the first three HMG boxes the sequences are $\geqslant 82 \%$ identical and $90 \%$ similar. The best match occurs in HMG box 1 which we have shown is responsible for most, if not all of the sequence-specific DNA binding of hUBF. The acidic carboxyl terminus is also highly similar. The region between the HMG boxes and the acidic tail, as well as the amino terminus are, less conserved. Interestingly, the part of the protein corresponding to most of human HMG box 4 (amino acids 388-474) is absent from frog UBF and probably accounts for the size difference be- 


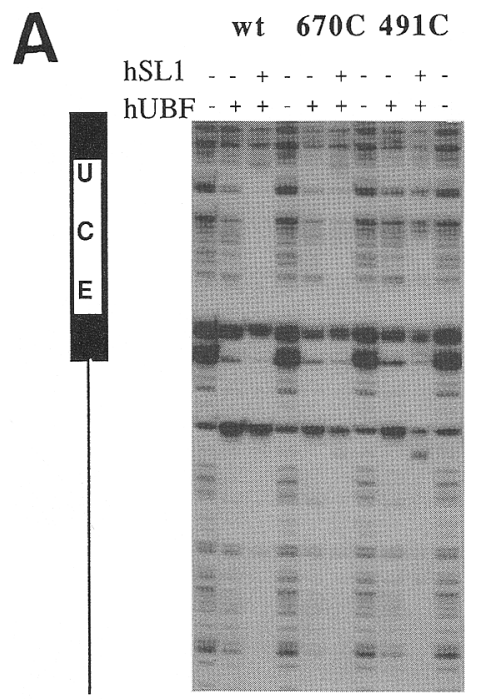

$1 \quad 2 \quad 3 \quad 4 \quad 5 \quad 6 \quad 7 \quad 8 \quad 910$
$367 \mathrm{C} 284 \mathrm{C} 204 \mathrm{C} 154 \mathrm{C}$

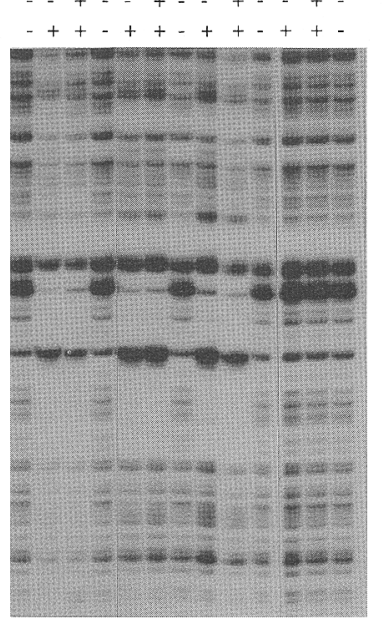

11121314151617181920212223
$84 \mathrm{~N} 182 \mathrm{~N} 284 \mathrm{~N}$

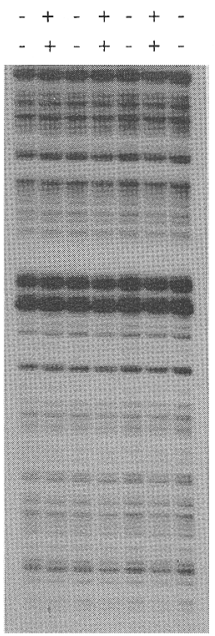

24252627282930
Pep84

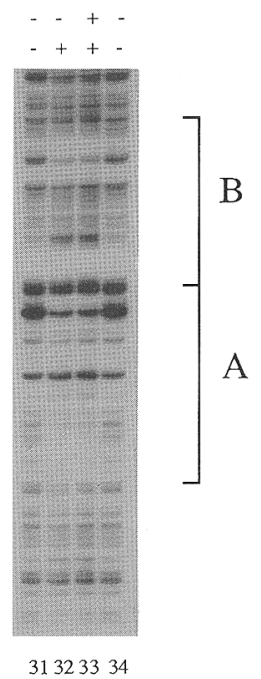

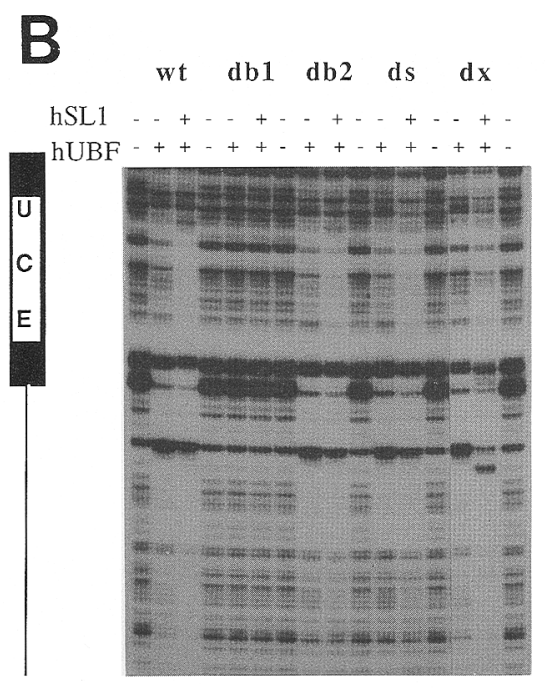

$1 \quad 2 \quad 3 \quad 4556788910111213141516$ db3 $\quad \mathrm{db} 4$

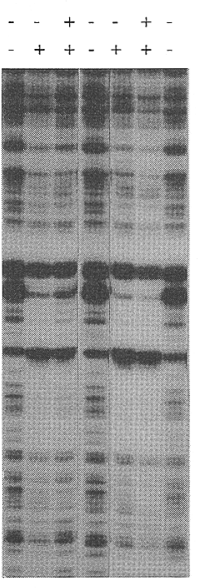

17181920212223

Figure 4. DNase I footprinting analysis of hUBF deletion mutants in the presence of hSLl. Deletion mutants of hUBF $(C)$ were translated in vitro and purified by DNA affinity chromatography. Maximal amounts of the proteins or HMG box peptide (Pep84) used in the DNase I protection experiments on the noncoding strand of the human rRNA promoter (Fig. 1A-C) were footprinted on the coding strand of the human ribosomal promoter in the absence or presence of $1 \mu \mathrm{g}$ of partially purified hSLl. The position of the UCE is shown at left; the brackets at right indicate regions protected from DNase I digestion by hUBF alone (A) or upon addition of both hUBF and hSLl (B). (A) Footprint analysis of carboxy- and amino-terminal deletion mutants and the HMG box peptide Pep84 in the presence of hSLl. $(B)$ Footprint analysis of internal deletion mutants in the presence of hSLl. $(C)$ Summary of the properties of hUBF deletion mutants. (Left) The structure of the deletion mutants is represented schematically. The position of the four HMG boxes and the acidic tail is shown at top. [Note that HMG box 4 was previously designated HMG box 3 (Jantzen et al. 1990); the additional HMG box is now box 3.] The ds mutant is identical to the naturally occurring splicing variant of hUBF resulting in an in-frame deletion of amino acids $221-257$ in the second HMG box and the $94-\mathrm{kD}$ form of the protein. (Right) Binding of the mutants translated in vitro to UBF-binding site DNA columns in the presence of nonspecific competitor is summarized (data not shown; see Results) and compared with their DNase I footprinting properties (derived from the data shown in Fig. 1A,B). The binding of hUBF mutants to DNA columns was scored as follows: $(++\mid \geqslant 50 \%$ of the protein was retained on the column at low salt concentration; $|+| 20-50 \%$ of the protein was retained. (n.d.) Not done. The results from the transcriptional activation experiments (Fig. 3A,B) and the data from the footprinting analysis in the presence of hSL1 (Fig. 4A,B) are summarized as follows: The 13- to 24-fold activation of the wild-type protein vs. the control from a mock translation was scored as ++++ ; an eightfold stimulation was scored +++ ; a four- to sixfold stimulation was ++ ; a 1.5- to threefold stimulation was + ; no detectable stimulation was - . A wild-type extension of the UBF footprint with hSL1 was indicated with ++ , whereas a shorter footprint extension was indicated with + . 
tween the two proteins. We have expressed xUBF by translation in vitro and verified that its binding specificity on the frog and human rRNA promoters is indistinguishable from $x U B F$ purified from $X$. laevis cells (kindly provided by M. Dunaway; A.M. Chow and H.-M. Jantzen, unpubl.). To demonstrate that the $\mathrm{xUBF}$ species we have cloned is functional for frog transcription, increasing amounts of in vitro-transcribed RNA encoding xUBF were coinjected into frog oocytes with a promoter-enhancer pseudo-wild-type construct (Dunaway 1989). Specific transcription in vivo of the reporter gene was stimulated in proportion to the amount of xUBF RNA injected (A.M. Chow and M. Dunaway, unpubl.).

Having confirmed the functional properties of our $x U B F$ cDNA clone in vivo, we then analyzed the transcriptional activity of $\mathrm{xUBF}$ in a reconstituted human in vitro transcription reaction. As shown previously for xUBF isolated from frog cells (Bell et al. 1989), no significant activation of transcription was observed when reconstituted with human Pol I and hSL1 (Fig. 5B, lanes 9-11). In addition, the DNase I footprint of xUBF in the presence of hSL1 displayed an altered pattern, indicative of a disrupted interaction with SLl (Fig 5C, lanes 9,10). Indeed, the altered pattern obtained with $\mathrm{xUBF}$ resembled the footprint detected with hUBF deletion mutants 670C, 491C, dx, and db4 (Fig. 4A, lanes 5,6,8,9; Fig 4B, lanes $14,15,21,22$, which are all defective in transcriptional activation. To determine the region of UBF responsible for the species specificity of transcription and cooperative DNA binding of hSL1, hybrid proteins were expressed after swapping the diverged carboxy-terminal portion of the proteins. Both the human/Xenopus and Xenopus/human hybrid (Fig. 5A) protected the human rRNA promoter from DNase I digestion in a manner indistinguishable from xUBF or hUBF (H.-M. Jantzen, unpubl.). The human/Xenopus hybrid that contains the first 341 amino acids from human UBF (including most of HMG box 3) fused to the carboxy-terminal amino acids of $x U B F$, retained only minimal transcriptional activity in the human in vitro transcription system, and thus behaved like full-length xUBF (Fig. 5B, lanes 12-14). As expected, the human/Xenopus hybrid, unlike UBF, leads only to a partial extension of the DNase I footprint in the presence of hSL1 (Fig. 5C, lanes 5, 6). In contrast, the Xenopus/human hybrid that contains the first 341 amino acids from xUBF fused to the carboxy-terminal amino acids of hUBF retained almost full transcriptional activity in the context of human Pol I and hSL1 (Fig. 5B, cf. lanes 15-17 with lanes 5-7). Moreover, the Xenopus/ human hybrid resulted in a fully extended DNase I footprint in the presence of hSLl, suggesting protein-protein interactions very similar to those with hUBF (Fig. 5C, cf. lanes 12 and 13 with lanes 2 and 3 ). These results strongly suggest that a major part of the species-specific behavior resides in a region of UBF overlapping $\mathrm{HMG}$ box 4 and the acidic tail. Furthermore, in contrast to deletions in the UBF protein that impair transcriptional activity, this gain of function by the Xenopus/human hybrid independently establishes the importance of the carboxy-terminal domains for mediating activation by
hUBF. These findings also underscore the critical importance of cooperative interactions between a site-specific activator, such as hUBF, and components of the TBPTAF complex in mediating transcription.

\section{Discussion}

In this paper we have addressed the critical relationship between activation domains of the RNA Pol I factor hUBF and its cooperative interactions with a TBP-TAF complex, hSL1. Several previous studies suggested interactions between pol II activators (USF, ATF, GAL4) and the TBP-containing TFIID fraction based on altered DNase I footprinting patterns, but their relation to specific activation domains remained obscure (Sawadogo and Roeder 1985; Horikoshi et al. 1988a,b). Here, we have demonstrated that HMG boxes 3 and 4 are required for strong interaction with the TBP-TAF complex hSLl, as suggested by the extended footprinting pattern. These findings also correlate well with the requirement of the two HMG boxes for full transcriptional activity. For example, a deletion of HMG box 3 simultaneously abolishes hSLl interaction and transcriptional activation (Fig. 6). This suggests the importance of activation domains in the interaction with components of the SL1 complex. Because direct interactions between the HMG boxes and specific components of hSL1 still remain to be demonstrated, we cannot exclude the possibility that these domains facilitate hSLl binding by changing the DNA structure rather than mediate protein-protein contacts. We favor, however, the simple model that hUBF makes direct contact with one or more components of hSLl to mediate activation.

One unique aspect of rRNA transcription is the species-specific nature of the promoter recognition process. We have therefore also studied the molecular basis for species specificity of hSL1 interactions with UBF from $X$. laevis, which cannot activate human Pol I transcription (Bell et al. 1989; Pikaard et al. 1989). Our results with human/Xenopus hybrid proteins suggest a strong correlation between altered protein-protein interactions with hSLl and reduced transcriptional activation. In addition we provide evidence for a crucial role of HMG box 4 and the acidic tail of hUBF in mediating cooperative DNA binding of hSL1.

Thus far hUBF is the only HMG box containing a transcription factor that has an acidic tail similar to that found in the chromosomal proteins HMG 1 and 2. Previously, we have proposed that this unusual domain might interact through negatively charged surfaces with hSL1 (Jantzen et al. 1990). The data reported in this paper not only confirm the critical role of this domain in interaction with hSL1 but also establish its strict requirement for strong transcriptional activation. These findings demonstrate that all domains of hUBF involved in potential interactions with the TBP-TAF complex hSLl are important for mediating transcriptional activation (Fig. 6). Similar, but less dramatic, effects have been reported for the acidic tail of $\mathrm{xUBF}$ on Xenopus transcription, although potential domains of interaction with 


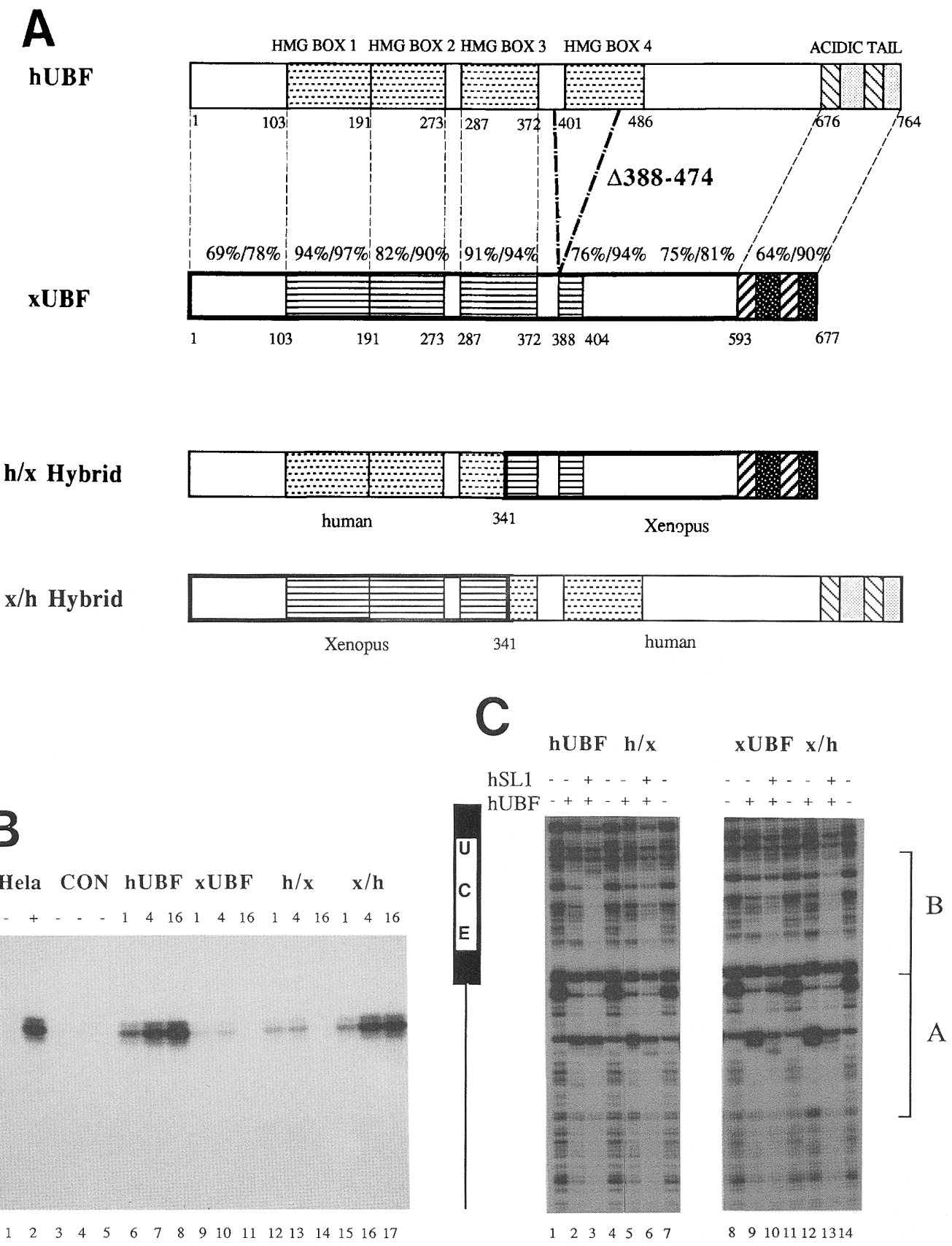

Figure 5. Properties of cloned $x U B F$ from $X$. laevis and of human/frog hybrid proteins. $(A)$ Schematic diagram of $x U B F$, hUBF, and human/frog hybrid proteins. (Top) A schematic representation of the alignment of the amino acid sequences of hUBF and xUBF. The positions of the HMG boxes and the acidic tail in the amino acid sequences are indicated by the numbers below the diagrams. Regions with amino acid similarity have been connected with interrupted lines, and the percentage of sequence identity and similarity are given above the xUBF representation. The "deletion" in xUBF homologous to amino acids $388-474$ in hUBF has been indicated. (Bottom) The structure of the human/Xenopus $(\mathrm{h} / \mathrm{x})$ and the Xenopus/human $(\mathrm{x} / \mathrm{h})$ hybrid proteins. The fusion has been made around amino acid 341 in HMG box 3. (B) Transcriptional activity of human/Xenopus hybrid proteins in the human Pol I system. Various amounts (as indicated above the lanes) of the affinity-purified UBF proteins (see Fig. 5A) were tested for transcriptional activation in vitro on a human rRNA promoter template with partially purified human Pol I and hSL1. Correctly initiated RNA products were detected by S1 nuclease analysis. As a positive control $0.5 \mathrm{ng}$ of hUBF purified from Hela cells was included (lane 2). As a negative control 1,2 , and $4 \mu \mathrm{l}$ of a mock translation reaction passed over an affinity column were assayed in the in vitro transcription system (lanes 3-5). (C) DNase I footprinting analysis of human/Xenopus $(h / x, x / h)$ hybrid proteins in the presence of hSL1. hUBF, $x U B F$, and the hybrid proteins $\mathrm{h} / \mathrm{x}$ and $\mathrm{x} / \mathrm{h}$ were footprinted on the coding strand of the human rRNA promoter in the absence or presence of 1 $\mu \mathrm{g}$ of partially purified hSL1, as indicated above the lanes. Amounts of the UBF proteins resulting in a full footprint on the noncoding strand of the promoter were used in all cases. Lanes 1,4,7,8,11, and 14 contain no protein. The position of the UCE is shown at left. Regions protected from DNase I digestion in the presence of UBF alone (A) or with both UBF and hSL1 (B) are bracketed at right. 
Figure 6. Functional domains of hUBF. In this diagram the four HMG boxes of hUBF are shown as shaded areas; the acidic carboxyl terminus is indicated by the hatched boxes. The proposed functional properties of the different regions of the molecule are summarized above and below with brackets.

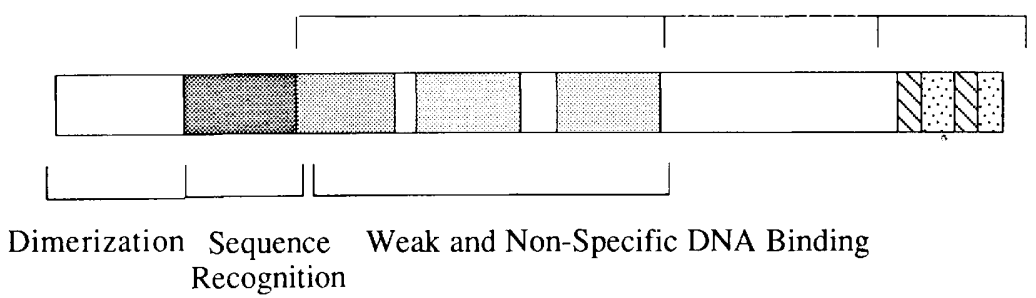

xSLl have not been demonstrated (McStay et al. 1991b). This underscores the importance of both the HMG boxes and the acidic domain for this transcriptional activator.

A surprising result of this study was the finding that not all transcriptional activation domains of hUBF necessarily interact with the TBP-TAF complex. Our findings suggest that $\mathrm{HMG}$ box 2 might direct interactions with another component of the initiation complex. Although interactions with the TBP-TAF complex seem to be crucial, they might not be sufficient. HMG box 2 might contact RNA Pol I directly or through factors associated with this enzyme. Alternatively, this domain might play a critical role in promoting the formation of the nucleoprotein superstructure of the initation complex (e.g., by DNA bending without directly contacting other proteins). It also remains possible that HMG box 2 interacts with hSL1, however, in a manner different from HMG boxes 3 and 4 that cannot be detected in the footprinting assay.

The array of multiple domains in hUBF with a dual role in DNA binding and protein-protein interaction is somewhat reminiscent of the POU domains of other transcription factors. Whereas the POU homeo domain determines most of the DNA-binding specificity, the POU-specific domain not only modulates the specificity but also strongly affects binding efficiency and is required for protein-protein interactions (Ingraham et al. 1990; Verrijzer et al. 1990). Curiously, there is $\sim 30 \%$ amino acid sequence similarity between the $B$ box of POU-specific domains and the central portion of HMG boxes, perhaps indicating similarity in function.

The unusual contribution of multiple domains to DNA-binding by hUBF could be explained by their potential role in altering the structure of the promoter DNA, as reported recently for other HMG domains (Lilley 1992). For example, DNA bending can be promoted by the HMG boxes of LEF-1, SRY, and mtTF1. In the case of LEF-1 the degree of bending is sufficient to facilitate communication between widely separated protein-binding sites (Giese et al. 1992). The potential of mtTF1 and ABF2, which both contain two HMG boxes, to generate negative supercoils in relaxed, double-stranded circular DNA is suggestive of the wrapping of the target DNA around the protein (Diffley and Stillman 1992; Fisher et al. 1992). Although these properties have yet to be verified for hUBF, the periodicity of enhanced DNase I cleavages on the Xenopus promoter in the presence of $\mathrm{XUBF}$ (designated TFIS) was proposed to be consistent with a wrapping of the DNA around xUBF (Dunaway 1989). In addition, prominently enhanced DNase I cleavages are located between conserved UBF-binding motifs in the core element and the UCE of the human rRNA promoter, which can be interpreted as the increased accessibility of the intervening sequences to DNase I owing to UBF-induced DNA bending. Interestingly, the HMG box proteins SSRP1/T160 and HMG 1, 2 have been shown to bind strongly to DNA modified by the anti-cancer compound cisplatin, which results in specific DNA bending and limited unwinding (Bruhn et al. 1992; Pil and Lippard 1992). Intriguingly, hUBF is efficiently recognized by cisplatin-modified DNA (D. Treiber, X. Zhai, and J. Essigmann, pers. comm.). The structure of hUBF with its multiple HMG boxes and the acidic tail might be ideal for mediating a combination of activities, including sequence-specific DNA interactions and possibly formation of a nucleoprotein superstructure involving DNA bending and wrapping required for productive interaction with the TBP-TAF complex hSL1 and RNA Pol I in the initiation complex.

\section{Materials and methods}

Construction of the expression vector pTBSTOP

The in vitro transcription-translation vector $\mathrm{pT} 7 \beta$ Sal [a pGEM4 derivative containing the $\beta$-globin gene leader downstream of the T7 promoter, provided by R. Treisman (Norman et al. 1988)] was modified to allow for the insertion and efficient expression of both amino- and carboxy-terminal deletion mutants and for excision of the expression cassette for further use in bacterial and eukaryotic vectors. The sequence $5^{\prime}$-CATGGCGCATATGTCGACAGGCCTAGCTAGCTAGCGGCCGC-3' was inserted between the NcoI and HindII sites of pT7ßSal using synthetic oligonucleotides resulting in pT $\beta$ STOP. The structure of the region of interest of pT $\beta$ STOP can be summarized as follows: T7 promoter, HindIII site, $\beta$-globin leader sequence, $N c o I$ (contains ATG), NdeI (ATG in-frame), SalI, StuI sites, three stop 
codons in three frames, NotI, XbaI, BamHI, SmaI, SacI, and EcoRI sites.

\section{Construction of hUBF deletion mutants}

The wild-type hUBF expression construct pT $\beta U B F 2$ was generated by ligating the NdeI-HindIII hUBF cDNA fragment of pSUBFINde (Jantzen et al. 1990) into the NdeI and StuI sites of pT $\beta S T O P$ after treating the 3' end of the fragment with Klenow enzyme. All constructs based on pT $\beta S T O P$ contain codons for an additional methionine, alanine, and histidine at the amino terminus of the insert owing to the ATG of the NcoI site. Amino-terminal deletion mutants ( $\mathrm{N}$ series) were generated by using restriction sites or exonuclease III and ligated in-frame with the ATG of the NdeI site into the Sall site. The overhanging nucleotides of the SalI site were either filled in with Klenow enzyme or removed with mung bean nuclease or, alternatively, the Sall site was cut with HindII to regenerate the reading frame. This procedure resulted in the insertion of the codons for the amino acids serine and proline after the ATG of the NdeI site of the mutants $84 \mathrm{~N}$ and $182 \mathrm{~N}$. The mutants $284 \mathrm{~N}$ and $491 \mathrm{~N}$ contain an insertion of the codon for serine, and $381 \mathrm{~N}$ contains an insertion of serine and threonine. The number in the designation of the amino-terminal mutant ( $\mathrm{N}$ series) refers to the position of the amino-terminal amino acid of hUBF retained in the mutant. Carboxy-terminal mutants of hUBF were constructed by using restriction sites, generating blunt ends, and ligating fragments abutting the StuI site $5^{\prime}$ of the three stop codons. The mutants $491 \mathrm{C}, 284 \mathrm{C}, 204 \mathrm{C}$, and $154 \mathrm{C}$ contain additional codons for proline and serine at the carboxyl terminus. Mutant $670 \mathrm{C}$ contains the additional sequence HQAPS /singleletter code) at the carboxyl terminus, whereas amino acid 367 of mutant $367 \mathrm{C}$ has been changed from a glutamic acid to an aspartic acid and the sequence LAS has been added. The mutant $90 \mathrm{C}$ was generated by TaqI digestion of $204 \mathrm{C}$. The number in the designation of the carboxy-terminal mutant (C series) refers to the last retained amino acid of hUBF.

Internal mutants (d series) were generated by using restriction sites of the hUBF cDNA, site-directed mutagenesis, and exonuclease III. The mutants were designated for the deletion of a certain HMG box $(\mathrm{dbl}-4)$ or the region between HMG box 4 and the acidic tail $(\mathrm{dx})$. The positions of the deleted or inserted amino acids of the mutants are as follows (see figures): $\mathrm{dbl}$; deletion of amino acids 101-181, insertion of $G, S, P$; db2; deletion of amino acids 205-284; db3, deletion of amino acids 284-371; db4, deletion of amino acids 371-491; dx, deletion of amino acids 492-670, insertion of proline.

The internal mutant ds represents a naturally occurring splicing variant of $h U B F$ resulting in the deletion of amino acids 221-257 within HMG box 2 and is expressed as the 94-kD form of the hUBF protein doublet (Chan et al. 1991; O'Mahoney and Rothblum 1991). For the insertion of this mutant into pTßSTOP, a BstXI-EcoRI fragment (positions 31 and 2310) of the plasmid pNOR5 (generously provided by Drs. E.K.L. Chan and E.M. Tan; Chan et al. 1991) was ligated into the BstXI site (position 171 of the hUBF sequence; Jantzen et al. 1990) and the EcoRI site at the 3 'end of the polylinker of pT $\beta S T O P$.

\section{Synthesis of HMG box peptides}

The peptides Pep84(103-186), Pep75(112-186), and Pep65(122186) spanning HMG box 1 of hUBF (positions of amino acids as indicated) were synthesized using solid-phase methodology (fluorenylmethoxycarbonyl amino acids activated by benzotriazolyltetramethylammonium hexafluorophosphate) on an ABI 431A peptide synthesizer. Cleavage and deprotection were ac- complished with reagent $\mathrm{K}$, and the crude peptides were purified to $>96 \%$ homogeneity by reverse-phase high-pressure liquid chromotography (HPLC) with a gradient of acetonitrile in water, both containing $0.1 \%$ trifluoroacetic acid. Structure and purity of the products were verified by electrospray ionizationmass spectroscopy. Circular dichroism and proton magnetic resonance spectra are consistent with a homogenous structure of the 84-mer peptide in solution (in collaboration with D. Wemmer, University of California, Berkeley).

\section{Cloning of cDNAs for X. laevis $U B F$}

A $\lambda$ gt10 cDNA library prepared from $X$. laevis stage 17-19 RNA (originally from D. Melton, via M. Kirschner, University of California, San Francisco) was screened with a $2-\mathrm{kb}$ fragment of hUBF cDNA (EcoRI-ApaLI site at position 2030; Jantzen et al. 1990) labeled using the random hexamer priming method. The experimental conditions were as described previously (Jantzen et al. 1990), except that the hybridization was performed at $48^{\circ} \mathrm{C}$ for $36 \mathrm{hr}$ and final washes were done at $50^{\circ} \mathrm{C}$ with $2 \times$ SST [SST: $150 \mathrm{~mm} \mathrm{NaCl}, 15 \mathrm{~mm}$ Tris, $2.5 \mathrm{~mm}$ EDTA (pH 7.5).] Twentyeight positives were obtained from $10^{6}$ clones screened, and four clones were analyzed further and partially sequenced. Three clones were overlapping with the clone xUBF1 /with internal EcoRI site) published by Bachvarov and Moss (1991), whereas one clone seemed to represent xUBF2 /without an internal EcoRI site; Bachvarov et al. 1991; McStay et al. 1991a). A representative clone of $\mathrm{xUBF} 1$, spanning $3.2 \mathrm{~kb}$ (from a SmaI site 224 nucleotides upstream of the first ATG to the EcoRI site derived from the $\lambda$ polylinker), was subcloned into the HindIII (after filling in with Klenow) and EcoRI sites of pBluescript KS + (Stratagene) resulting in pKxUBF2B. The insert was sequenced completely on both strands using exonuclease-generated deletion mutants and Sequenase reagents (U. S. Biochemical).

\section{Construction of Xenopus UBF and human-frog hybrid expression plasmids}

For expression by transcription-translation in vitro the ATG start codon sequence of the $\mathrm{xUBF}$ cDNA was converted into a NdeI site by site-directed mutagenesis and the $2.3-\mathrm{kb}$ NdeI-ScaI $(0.27 \mathrm{~kb}$ downstream of the stop codon) fragment was cloned into the NdeI and StuI sites of pT $\beta S T O P$, resulting in the expression vector $\mathrm{p} T \beta \times \mathrm{XUB}$.

The human-frog hybrid expression vector $\mathrm{pT} \beta \mathrm{h} / \mathrm{x} 34 \mathrm{l}$ was generated by fusion of the human cDNA fragment encoding the first 341 amino acids at the $B s a H I$ site to a frog cDNA fragment encoding amino acids 341 (common $B s a \mathrm{HI}$ site) to 677 and including the frog $3^{\prime}$-untranslated region to the Scal site. The frog-human hybrid expression vector $\mathrm{pT} \beta \mathrm{x} / \mathrm{h} 341$ was constructed using the $5^{\prime}$ fragment from xUBF1 (NdeI-BsaHI) and the 3 ' fragment from hUBF cDNAs (BsaHI-HindIII). These fragments were ligated into the vector pT $\beta S T O P$ (NdeI and StuI sites).

\section{In vitro translation and purification of UBF proteins}

Expression vectors containing the various hUBF and $x U B F$ inserts were linearized and transcribed in vitro with T7 RNA polymerase for $2 \mathrm{hr}$. T7 RNAs produced from $3.2 \mu \mathrm{g}$ of DNA template were translated in vitro in a $0.4-\mathrm{ml}$ reaction using 1.4 $\mu \mathrm{M}\left[{ }^{35} \mathrm{~S}\right]$ methionine $(90 \mathrm{Ci} / \mathrm{mmole})$ with rabbit reticulocyte lysates (Promega). UBF proteins were partially purified by sequence-specific DNA affinity chromatography using $30 \mu \mathrm{l}$ of a matrix containing the UBF-binding site of the human UCE (Bell et al. 1988). Before loading, $40 \mu \mathrm{g}$ of poly[d(AT)]/[d(AT)] and $1 \mu \mathrm{g}$ 
of salmon sperm competitor DNA, $0.2 \mathrm{ml}$ of $\mathrm{TM}^{+} 0.1$ and $0.8 \mathrm{ml}$ of $\mathrm{TM}^{+} 0$, were added to the translation reaction $\left[\mathrm{TM}^{+}: 50 \mathrm{mM}\right.$ Tris- $\mathrm{HCl}$ ( $\mathrm{pH} 7.9$ ), $12.5 \mathrm{mM} \mathrm{MgCl}_{2}, 20 \%$ glycerol, $0.5 \mathrm{~mm}$ EDTA, $1 \mathrm{mM}$ DTT, $0.1 \% \mathrm{NP}-40$, and $\mathrm{KCl}$ as indicated]. After loading twice, the columns were washed with a total of $0.45 \mathrm{ml}$ of $\mathrm{TM}^{+} 0.1$. Most of the UBF protein was recovered in a $75-\mu$ l fraction by elution with $\mathrm{TM}^{+} 0.6$. To adjust for differences in expression and recovery of the different proteins, aliquots were analyzed by SDS-PAGE ( $8 \%$ acrylamide), and autoradiographed, and the radioactive protein bands were excised and quantified by liquid scintillation counting. The amounts of all proteins were normalized to in vitro-translated full-length hUBF and corrected for the number of methionines in each protein to allow for the use of equimolar amounts in functional experiments.

\section{DNA-binding and in vitro transcription assays}

The binding of hUBF deletion mutants to DNA affinity columns bearing the UBF-binding site of the human promoter upstream control element was analyzed in the presence of nonspecific competitor essentially as described (Jantzen et al. 1990). DNase I footprinting reactions were performed as reported previously (Jantzen et al. 1990), except that calcium chloride was omitted and the DNase I concentration was raised $\sim 100$-fold whenever in vitro-translated material was used. Conditions for in vitro transcription and nuclease $S 1$ assays were identical to those used previously (Jantzen et al. 1990). The radioactivity in the gel corresponding to correctly initiated transcripts was quantified using the PhosphorImager (Molecular Dynamics).

\section{Chemical cross-linking experiments}

hUBF proteins translated in vitro in the presence of $\left[{ }^{35} \mathrm{~S}\right]$ methionine and partially purified by sequence-specific affinity chromatography were incubated with or without $5 \mathrm{~mm}$ EGS [ethylene glycol-bis(succinic acid- $N$-hydroxysuccinimide ester), Sigma] in $0.1 \mathrm{M}$ triethanolamine (pH8), $5 \%$ glycerol, and $2 \mathrm{~mm}$ EDTA for $15 \mathrm{~min}$ at room temperature. The reactions were terminated by addition of lysine at a final concentration of $20 \mathrm{~mm}$. The products were separated by SDS-PAGE ( $8 \%$ acrylamide) and autoradiographed.

\section{Acknowledgments}

We thank C. Altmann and S. Bell for their help in cloning xUBF cDNAs, M. Dunaway for Xenopus oocyte injections, valuable reagents, and helpful discussions. The generosity of E.K.L. Chan, E.M. Tan, and T. Moss in sharing materials or information before publication is especially appreciated. S. Bell and L. Comai provided part of the hSL1 and hPol I preparations. We thank members of the Tjian, Dunaway, Harland, and Botchan laboratories for helpful advice and support. We are grateful to $\mathrm{L}$. Comai, M. Dunaway, B. Dynlacht, G. Gill, and N. Tanese for critically reading the manuscript, and to $\mathrm{K}$. Ronan for help during its preparation. H.-M.J. was supported in part by a postdoctoral fellowship from the American Cancer Society, California Division (S-41-89). R.T. was supported in part by a grant from the National Cancer Institute (CA 25417).

\section{References}

Bachvarov, D. and T. Moss. 1991. The RNA Pol I transcription factor XUBF contains 5 tandemly repeated HMG homology boxes. Nucleic Acids Res. 19: 2331-2335.
Bachvarov, D., M. Normandeau, and T. Moss. 1991. Heterogeneity in the Xenopus ribosomal transcription factor xUBF has a molecular basis distinct from that in mammals. FEBS Lett. 288: 55-59.

Bell, S.P., R.M. Learned, H.-M. Jantzen, and R. Tjian. 1988. Functional cooperativity between transcription factors UBFl and SLl mediates human ribosomal RNA synthesis. Science 241: 1192-1197.

Bell, S.P., C.S. Pikaard, R.H. Reeder, and R. Tjian. 1989. Molecular mechanisms governing species-specific transcription of ribosomal RNA. Cell 59: 489-497.

Bell, S.P., H.-M. Jantzen, and R. Tjian. 1990. Assembly of alternative multiprotein complexes directs rRNA promoter selectivity. Genes \& Dev. 4: 943-954.

Bruhn, S.L., P.M. Pil, J.M. Essigmann, D.E. Housman, and S. J. Lippard. 1992. Isolation and characterization of human cDNA clones encoding a high mobility group box protein that recognizes structural distortions to DNA caused by binding of the anticancer agent cisplatin. Proc. Natl. Acad. Sci. 89: 2307-2311.

Chan, E.K.L., H. Imai, J.C. Hamel, and E.M. Tan. 1991. Human autoantibody to RNA Pol I transcription factor hUBF. Molecular identity of nucleolus organizer region autoantigen NOR-90 and ribosomal RNA transcription upstream binding factor. J. Exp. Med. 174: 1239-1244.

Comai, L., N. Tanese, and R. Tjian. 1992. The TATA-binding protein and associated factors are integral components of the RNA Pol I transcription factor, SL1. Cell 68: 965-976.

Cormack, B.P. and K. Struhl. 1992. The TATA-binding protein is required for transcription by all three nuclear RNA polymerases in yeast cells. Cell 69: 685-696.

Diffley, J.F.X. and B. Stillman. 1991. A close relative of the nuclear, chromosomal high-mobility group protein HMG1 in yeast mitochondria. Proc. Natl. Acad. Sci. 88: 7864-7868.

1992. DNA binding properties of an HMGl-related protein from yeast mitochondria. I. Biol. Chem. 267: 33683374.

Dunaway, M. 1989. A transcription factor, TFIS, interacts with both the promoter and enhancer of the Xenopus rRNA genes. Genes \& Dev. 3: 1768-1778.

Dynlacht, B.D., T. Hoey, and R. Tjian. 1991. Isolation of coactivators associated with the TATA-binding protein that mediate transcriptional activation. Cell 66: 563-576.

Fisher, R.P., T. Lisowsky, M.A. Parisi, and D.A. Clayton. 1992. DNA wrapping and bending by a mitochondrtial high mobility group-like transcriptional activator protein. $J$. Biol. Chem. 267: 3358-3367.

Giese, K., J. Cox, and R. Grosschedl. 1992. The HMG domain of lymphoid enhancer factor 1 bends DNA and facilitates assembly of functional nucleoprotein structures. Cell 69: 185195.

Gill, G. and R. Tjian. 1992. Eukaryotic coactivators associated with the TATA box binding protein. Curr. Opin. Genet. Dev. 2: 236-242.

Greenblatt, J. 1991. Roles of TFID in transcriptional initiation by RNA Pol II. Cell 66: 1067-1070.

Gubbay, J., J. Collignon, P. Koopman, B. Capel, A. Economu, A. Munsterberg, N. Vivian, P. Goodfellow, and R. Lovell-Badge. 1990. A gene mapping to the sex-determining region of the mouse $\mathrm{Y}$ chromosome is a member of a novel family of embryonically expressed genes. Nature 346: 245-250.

Hisatake, K., T. Nishimura, Y. Maeda, K. Hanada, C. Song, and M. Muramatsu. 1991. Cloning and structural analysis of cDNA and the gene for mouse transcription factor UBF. Nucleic Acids Res. 19: 4631-4637.

Horikoshi, M., M.F. Carey, H. Kakidani, and R.G. Roeder. 
1988a. Mechanism of action of a yeast activator: Direct effect of GAL4 derivatives on mammalian TFIID-promoter interactions. Cell 54: 665-669.

Horikoshi, M., T. Hai, Y. Lin, M.R. Green, and R.G. Roeder. 1988b. Transcription factor ATF interacts with the TATA factor to facilitate establishment of a preinitiation complex. Cell 54: 1033-1042.

Ingraham, H.A., S.E. Flynn, J.W. Voss, V.R. Albert, M.S. Kapiloff, L. Wilson, and M.G. Rosenfeld. 1990. The POU-specific domain of pit- 1 is essential for sequence-specific, high-affinity DNA-binding and DNA-dependent pit-1-pit-1 interactions. Cell 61: 1021-1033.

Jantzen, H.-M., A. Admon, S.P. Bell, and R. Tjian. 1990. Nucleolar transcription factor hUBF contains a DNA-binding motif with homology to HMG proteins. Nature 344: 830-836.

Johnson, P.F. and S.L. McKnight. 1989. Eukaryotic transcriptional regulatory proteins. Annu. Rev. Biochem. 58: 799839.

Learned, R.M., T.K. Learned, M.M. Haltiner, and R.T. Tjian. 1986. Human rRNA transcription is modulated by the coordinate binding of two factors to an upstream control element. Cell 45: 847-857.

Lilley, D.M.J. 1992. HMG has DNA wrapped up. Nature 357: 282-283.

Lobo, S.M., J. Lister, M.L. Sullivan, and N. Hernandez. 1991. The cloned RNA Pol II transcription factor IID selects RNA Pol III to transcribe the human U6 gene in vitro. Genes \& Dev. 5: 1477-1489.

Margottin, F., G. Dujardin, M. Gerard, J.-M. Egly, J. Huet, and A. Sentenac. 1991. Participation of the TATA factor in transcription of the yeast U6 gene by RNA polymerase C. Science 251: 424-426.

McStay, B., C.H. Hu, C.S. Pikaard, and R.H. Reeder. 1991 a. $\mathrm{xUBF}$ and Ribl are both required for formation of a stable polymerase I promoter complex in $X$. laevis. EMBO $I$. 10: $2297-2303$.

McStay, B., M.W. Frazier, and R.H. Reeder. 1991b. xUBF contains a novel dimerization domain essential for RNA Pol I transcription. Genes \& Dev. 5: 1957-1968.

Mitchell, P.J. and R. Tjian. 1989. Transcriptional regulation in mammalian cells by sequence-specific DNA-binding proteins. Science 245: 371-378.

Norman, C., M. Runswick, R. Pollock, and R. Treisman. 1988. Isolation and properties of cDNA clones encoding SRF, a transcription factor that binds to the $\mathrm{c}$-fos serum response element. Cell 55: 989-1003.

O'Mahoney, D.J. and L.I. Rothblum. 1991. Identification of two forms of the RNA Pol I transcription factor UBF. Proc. Natl. Acad. Sci. 88: 3180-3184.

Parisi, M.A. and D.A. Clayton. 1991. Similarity of human mitochondrial transcription factor 1 to high mobility group proteins. Science 252: 965-969.

Pikaard, C.S., B. McStay, M.C. Schultz, S.P. Bell, and R.H. Reeder. 1989. The Xenopus ribosomal gene enhancers bind an essential polymerase I transcription factor, xUBF. Genes \& Dev. 3: 1779-1788.

Pil, P.M. and S.J. Lippard. 1992. Specific binding of chromosomal protein HMG 1 to DNA damaged by the anticancer drug cisplatin. Science 256: 234-237.

Pugh, B.F. and R. Tiian. 1990. Mechanism of transcriptional activation by Spl: Evidence for coactivators. Cell 61: 11871197.

- 1991. Transcription from a TATA-less promoter requires a multisubunit TFIID complex. Genes \& Dev. 5: 1935-1945.

—_ 1992. Diverse transcriptional functions of the multisub- unit eukaryotic TFIID complex. I. Biol. Chem. 267: 679682.

Reeder, R.H. 1990. rRNA synthesis in the nucleolus. Trends Genet. 6: 390-395.

Roeder, R.G. 1991. The complexities of eukaryotic transcription initiation: regulation of preinitiation complex assembly. Trends Biochem. 16: 402-408.

Sawadogo, M. and R.G. Roeder. 1985. Interaction of a genespecific transcription factor with the adenovirus major late promoter upstream of the TATA box region. Cell 43: 165175.

Sawadogo, M. and A. Sentenac. 1990. RNA polymerase B (II) and general transcription factors. Annu. Rev. Biochem. 59: 711754.

Schultz, M.C., R.H. Reeder, and S. Hahn. 1992. Variants of the TATA-binding protein can distinguish subsets of RNA Pol I, II, and III promoters. Cell 69: 697-702.

Simmen, K.A., J. Bernues, H.D. Parry, H.G. Stunnenberg, A. Berkenstam, B. Cavallini, J.-M. Egly, and I.W. Mattaj. 1991. TFIID is required for in vitro transcription of the human U6 gene by RNA Pol III. EMBO J. 10: 1853-1862.

Sinclair, A.M., P. Berta, M.S. Palmer, J.R. Hawkins, B.L. Griffiths, D.J. Smith, J.W. Foster, A.-M. Frischauf, R. LovellBadge, and P.N. Goodfellow. 1990. A gene from the human sex-determining region encodes a protein with homology to a conserved DNA-binding motif. Nature 346: 240-244.

Smith, S.D., E. Oriahi, D. Lowe, H.-F. Yang-Yen, D. O'Mahoney, K. Rose, K. Chen, and L. I. Rothblum. 1990. Characterization of factors that direct transcription of rat ribosomal DNA. Mol. Cell. Biol. 10: 3105-3116.

Sollner-Webb, B. and E. B. Mougey. 1991. News from the nucleolus. Trends Biochem. 16: 58-62.

Tanese, N., B.F. Pugh, and R. Tiian. 1991. Coactivators for a proline-rich activator purified from the multisubunit TFIID complex. Genes \& Dev. 5: 2212-2224.

Travis, A., A. Amsterdam, C. Belanger, and R. Grosschedl. 1991. LEF-1, a gene encoding a lymphoid-specific protein with an HMG domain, regulates T-cell receptor $\alpha$ function. Genes \& Dev. 5: 880-894.

van de Wetering, M., M. Oosterwegel, D. Dooijes, and H. Clevers. 1991. Identification and cloning of TCF-1, a lymphoidspecific transcription factor containing a sequence-specific HMG box. EMBO I. 10: 123-132.

Verrijzer, C.P., A.J. Kal, and P.C. van der Vliet. 1990. The oct-1 homeo domain contacts only part of the octamer sequence and full Oct-1 DNA-binding activity requires the POU-specific domain. Genes \& Dev. 4: 1964-1974.

Waterman, M.L., W.H. Fischer, and K.A. Jones. 1991. A thymusspecific member of the HMG protein family regulates the human T-cell receptor $\alpha$ enhancer. Genes \& Dev. 5: 656669.

White, R.J., S.P. Jackson, and P.W.J. Rigby. 1992. A role for the TATA-box-binding protein component of the transcription factor IID complex as a general RNA Pol III transcription factor. Proc. Nat1. Acad. Sci. 89: 1949-1953. 


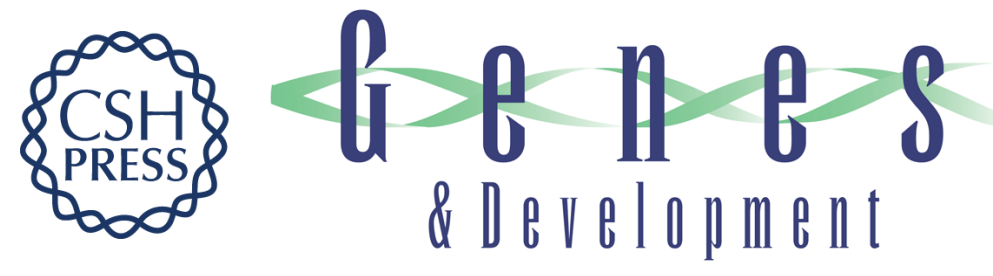

\section{Multiple domains of the RNA polymerase I activator hUBF interact with the TATA-binding protein complex hSL1 to mediate transcription.}

H M Jantzen, A M Chow, D S King, et al.

Genes Dev. 1992, 6:

Access the most recent version at doi:10.1101/gad.6.10.1950

References This article cites 53 articles, 24 of which can be accessed free at: http://genesdev.cshlp.org/content/6/10/1950.full.html\#ref-list-1

License

Email Alerting Service

Receive free email alerts when new articles cite this article - sign up in the box at the top right corner of the article or click here.

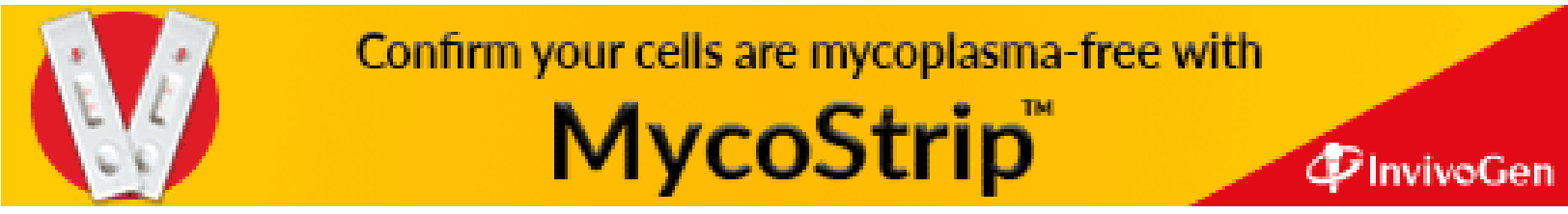

